\title{
The effects of recombinant human growth hormone therapy on thyroid function in pediatric patients with growth hormone deficiency
}

\author{
Qian Yao $^{1 \#}$, Dong Zheng ${ }^{2 \#}$, Yan Liang ${ }^{1}$, Ling Hou ${ }^{1}$, Yan-Qin Ying ${ }^{1}$, Xiao-Ping Luo ${ }^{1}$, Wei Wu ${ }^{1}$ \\ ${ }^{1}$ Department of Pediatrics, Tongji Hospital, Tongji Medical College, Huazhong University of Science and Technology, Wuhan, China; ${ }^{2}$ Department \\ of Orthopaedics, Union Hospital, Tongji Medical College, Huazhong University of Science and Technology, Wuhan, China \\ Contributions: (I) Conception and design: Q Yao, D Zheng; (II) Administrative support: Wei Wu; (III) Provision of study materials or patients: \\ Y Liang, L Hou; (IV) Collection and assembly of data: YQ Ying, XP Luo; (V) Data analysis and interpretation: YQ Ying, XP Luo; (VI) Manuscript \\ writing: All authors; (VII) Final approval of manuscript: All authors. \\ \#These authors contributed equally to this work. \\ Correspondence to: Wei Wu. Department of Pediatrics, Tongji Hospital, Tongji Medical College, Huazhong University of Science and Technology, \\ No. 1095 of Jiefang Avenue, Qiaokou District, Wuhan 430030, China. Email: wuw652147@163.com.
}

Background: Recombinant human growth hormone (rhGH) was approved for the therapy of pediatric patients with growth hormone deficiency (GHD) by the Food and Drug Administration (FDA) of the United States in 1985. This study aims to evaluate the effects of rhGH therapy on thyroid function in pediatric patients with GHD.

Methods: A total of 55 pediatric patients, who had been diagnosed with GHD and received rhGH therapy for 6-24 months, and who could regularly come to our hospital for outpatient visits from May 1, 2014 to April 30, 2017, were selected for the study. All of the patients were treated for at least six months, among which 44 patients were treated for 12 months, and 32 patients were treated for 18 months, and 16 patients were treated for 24 months.

Results: (I) During the course of the rhGH therapy, none of the patients had a free thyroxine (FT4) level lower than the normal lower limit. (II) The FT4 level decreased during the course of the therapy, when compared to the level at baseline, and the difference was statistically significant after 24 months of therapy. In the puberty group, the FT4 level had significantly decreased by the $12^{\text {th }}$ month of therapy, when compared to the baseline, but there was no significant change in the FT4 and thyroid-stimulating hormone (TSH) levels at the remaining observation time points of treatment.

Conclusions: Growth hormone (GH) replacement therapy may affect the metabolism of the thyroid hormone in pediatric patients with GHD. During the course of treatment, the changes in thyroid function in pediatric patients with GHD should be regularly monitored in order to identify any abnormal thyroid function in its early stages.

Keywords: Growth hormone deficiency; puberty status; recombinant human growth hormone therapy; thyroid function; thyrotropin; free thyroxine

Submitted Nov 24, 2020. Accepted for publication Jan 29, 2021.

doi: $10.21037 /$ tp-20-401

View this article at: http://dx.doi.org/10.21037/tp-20-401 


\section{Introduction}

Recombinant human growth hormone (rhGH) was approved for the therapy of pediatric patients with GHD by the Food and Drug Administration (FDA) of the United States in 1985 . The main goal of this therapy is to increase the height of pediatric and adolescent patients and get their final height as close as possible to their genetic target height. Its effectiveness and safety have been comprehensively evaluated $(1,2)$ and widely recognized. However, the dose of the GH therapy has to be individualized since the age, bone age, and height of patients with GHD at the beginning of therapy are closely correlated to their expected height in adulthood and, thus, affects the necessary duration of the therapy.

The organs in the endocrine system can interact with each other. It has been reported that the axis of GH/ insulin-like growth factor 1 (IGF-1) and the axis of the hypothalamus pituitary thyroid (HPT) are interrelated, and that the secretion and effect of IGF-1, IGF binding protein 3 (IGFBP-3) and GH are influenced by the axis of the HPT $(3,4)$. Furthermore, the GH/IGF-1 axis can change the growth and function of the thyroid gland and lead to changes in thyroid hormone metabolism in peripheral tissues (3-5).

However, at present, there are differences in the results of experimental and clinical studies concerning the effects of rhGH therapy on thyroid function. Notably, emerging lines of evidence have shown that there is a relationship between $\mathrm{GH}$ and the hypothalamic-pituitary-thyroid axis $(3,6)$. It has also been reported that the GH/IGF-1 axis could change the function of the thyroid gland and alter the metabolism of thyroid hormone in peripheral tissues (4). A study by Akin et al. (7) highlighted that GH therapy in children with idiopathic GHD does not induce central hypothyroidism, but in children with multiple pituitary hormone deficiencies, GH therapy usually shows up the presence of central hypothyroidism.

GH replacement therapy appears to have a controversial effect on thyroid function in pediatric patients with GHD, and the majority of studies have only followed up patients for 3-12 months after GH therapy has begun. Thus, the present study aims to observe the changes in thyroid function in pediatric patients with GHD during GH replacement therapy by conducting a retrospective analysis of the case data of pediatric patients with GHD in our hospital.

We present the following article in accordance with the
MDAR checklist (available at http://dx.doi.org/10.21037/ tp-20-401).

\section{Methods}

\section{Study subjects}

Pediatric patients who were admitted to the Pediatric Endocrine Ward of our hospital and diagnosed as GHD, between May 2014 and April 2017, were enrolled in the present study. The study was conducted in accordance with the Declaration of Helsinki (as revised in 2013). The study was approved by the Ethics Committee of Tongji Hospital (NO.: 2014-120) and informed consent was taken from all the patients.

The inclusion criteria were as follows: (I) patients whose height was lower than the average height of normal children of the same age and same gender by two standard deviations, or below the $3^{\text {rd }}$ percentile at the time of the visit (the average height and standard deviation of normal children of the same age and same gender was based on the height data in a physical development survey of children 0-18 years of age in nine cities in China in 2005); (II) patients with growth retardation and an annual growth rate of $<5 \mathrm{~cm} /$ year; (III) patients with symmetrical short stature but normal intellectual development; (IV) patients with a peak serum GH level of $<10 \mathrm{ng} / \mathrm{mL}$ in the two drug (insulin and clonidine) GH stimulation test; (V) patients with a bone age two years lower than their real age; (VI) patients of either gender who were 6-15 years of age at the time of the visit; (VII) patients with normal blood glucose regulation function i.e., fasting blood glucose $<5.6 \mathrm{mmol} / \mathrm{L}$ and postprandial blood glucose $<7.8 \mathrm{mmol} / \mathrm{L}$; and, (VIII) patients who had never received GH therapy before visiting our hospital.

The exclusion criteria were as follows: (I) patients with multiple pituitary hormone deficiencies; (II) patients with hepatic and renal dysfunction (ALT >2 times the normal upper limit, and $\mathrm{Cr}$ greater than the normal upper limit); (III) patients positive for anti-HBc, HBsAg and $\mathrm{HBeAg}$ in the viral detection of hepatitis B; (IV) patients with diabetes mellitus or with organic diseases including severe cardiac, pulmonary, and renal diseases, with blood system diseases, malignant tumors, or with systemic infection, low immune function, and nutritional metabolic disorders; (V) patients with other types of growth and developmental abnormalities, such as Turner syndrome, hypothyroidism, and other genetic metabolic diseases, and bone development 
Table 1 The items of the clinical data in the present study

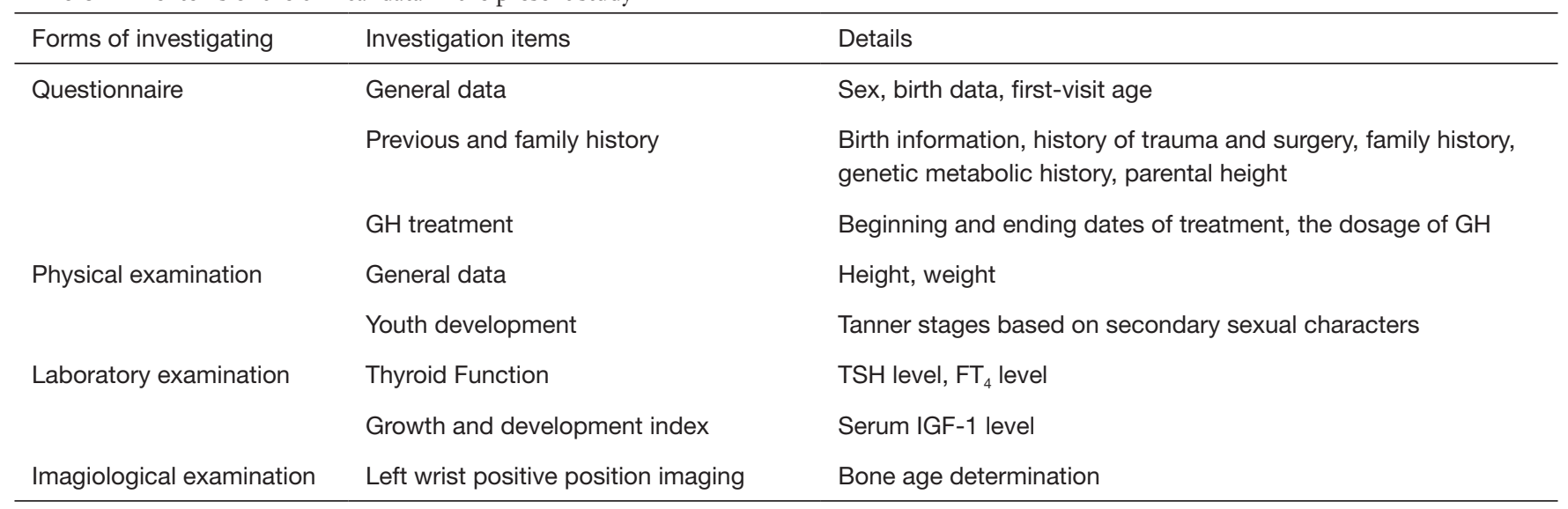

disorders.

According to the above inclusion and exclusion criteria, 55 pediatric patients with GHD were included in our retrospective analysis of the effects of rhGH therapy on thyroid function. All of the patients were treated for at least six months, 44 were treated for 12 months, 32 were treated for 18 months, and 16 patients were treated for 24 months. In terms of gender, 37 patients were male and 18 were female. The male-to-female ratio was, thus, 2.06:1.00. Their average age was $8.99 \pm 2.43$ years old, and 44 of them were assigned to the pre-puberty group, while the remaining 11 patients were assigned to the puberty group.

\section{Data collection}

All the pediatric patients were required to have a comprehensive physical examination and related laboratory tests every 2-3 months after the first visit, or from the beginning of the rhGH therapy. The physical examinations included the measurement of height and weight using the same tool and performed by a specially assigned staff member (taking the average value of three measurements), the recording of the growth rate, and the evaluation of the development of secondary sexual characteristics using Tanner's staging. A routine blood test was done, and hepatic and renal function and levels of thyroid hormone and serum IGF-1 were examined under fasting conditions for all patients. A left wrist $\mathrm{X}$-ray was taken every six months to one year in order to evaluate the bone age before and after the rhGH therapy. On the first visit, related laboratory examinations also included the two-drug (insulin and clonidine) stimulation test of GH as well as imaging tests, such as the analysis of bone age, and pituitary MRI examinations, to determine whether a pituitary disease was present. The relevant clinical data are presented in Table 1 .

\section{Detection of serum thyroid hormone levels}

The detection of thyroid hormone was performed as follows: $2 \mathrm{~mL}$ of venous blood was collected before the patient had eaten in the morning, and the serum was separated. Then, the TSH and FT4 levels were measured by chemiluminescent immunoassay. Following the manufacturer's instructions for SIEMENS IMMULITE2000 Total T4 and BECKMAN COULTER Access TSH, we detected the concentration of T4 and TSH using the IMMULITE 2000 XPi system. According to the reference limit of thyroid hormone in the Pediatric Metabolism Laboratory of our hospital, the normal range of FT4 was $0.8-1.9 \mathrm{ng} / \mathrm{dL}$, while the normal range of TSH was $0.6-4.5 \mathrm{mIU} / \mathrm{L}$.

\section{Evaluation methods}

(I) The thyroid hormone levels (TSH and FT4) in the 55 pediatric patients with GHD were recorded before therapy, and at $6,12,18$, and 24 months after rhGH therapy had begun and any changes observed. (II) The 55 pediatric patients with GHD were divided into two groups, according to their puberty state before treatment: a puberty group and a pre-puberty group. The changes in the level of thyroid hormone in these two groups were observed during the treatment. 
Table 2 The changes in TSH and FT4 levels in pediatric patients with GHD at baseline, and at 6, 12, 18 and 24 months after rhGH replacement therapy $(\bar{x} \pm \mathrm{SD})$

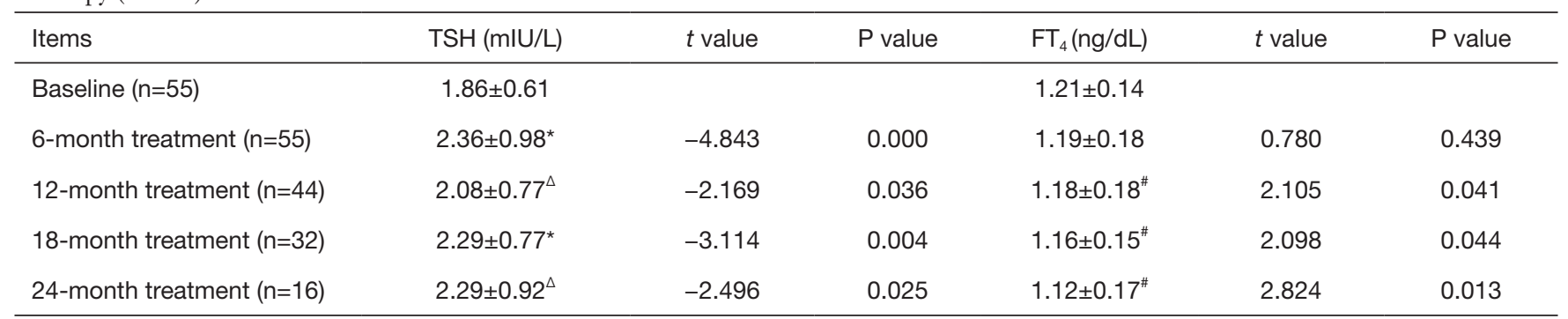

Compared with the baseline, ${ }^{*} \mathrm{TSH}$ level increased after rhGH treatment, $\mathrm{P}<0.01$; compared with the baseline, ${ }^{\Delta} \mathrm{TSH}$ level increased after rhGH treatment, $\mathrm{P}<0.05$; compared with the baseline, ${ }^{*} \mathrm{FT}_{4}$ level after $\mathrm{rhGH}$ treatment decreased, $\mathrm{P}<0.05$.

\section{Statistic analysis}

SPSS 19.0 software was used to perform a statistical analysis of the data collected. The measurement data, which included the demographic indicators and laboratory test indicators, were expressed as mean \pm standard deviation $(\bar{x} \pm \mathrm{SD})$. Variance analysis, Pearson correlation analysis, multiple linear regression analysis, a chi square test, a $t$-test, and other statistical methods were used to analyze the data. $P<0.05$ was considered statistically significant.

\section{Results}

Changes in thyroid function before and after the commencement of rbGH therapy in pediatric patients with GHD

A total of 55 pediatric patients with good compliance (patients who could regularly visit our hospital, and had complete follow-up data), who were diagnosed with GHD and received rhGH therapy in our hospital, were included in the present study. The average therapeutic dosage of $\mathrm{rhGH}$ was $0.16 \pm 0.014 \mathrm{U} / \mathrm{kg} \cdot \mathrm{d}$, which was administered subcutaneously 30 minutes before bedtime every night. The changes in TSH and FT4 levels in pediatric patients with GHD at baseline and during the GH replacement therapy are presented in Table 2.

During the course of the rhGH therapy, none of the pediatric patients had a FT4 level lower than the lower limit of a normal TSH. Three pediatric patients in the prepuberty group had a transient TSH value higher than the upper limit of a normal TSH at the $6^{\text {th }}, 12^{\text {th }}$ and $18^{\text {th }}$ month, but this returned to the normal level.

Compared with the baseline value of $1.86 \pm 0.61 \mathrm{mIU} / \mathrm{L}$, the TSH level of $2.36 \pm 0.98 \mathrm{mIU} / \mathrm{L}$ was significantly higher at six months after the rhGH therapy, and the difference was statistically significant $(\mathrm{P}=0.000)$. Furthermore, the TSH level at the $12^{\text {th }}, 18^{\text {th }}$ and $24^{\text {th }}$ month was $2.08 \pm 0.77$, $2.29 \pm 0.77$ and $2.29 \pm 0.92 \mathrm{mIU} / \mathrm{L}$, respectively, which was still higher than that at baseline, and the difference was statistically significant $(\mathrm{P}=0.036, \mathrm{P}=0.004$ and $\mathrm{P}=0.025$, respectively). When comparing the levels of FT4, it was found that after six months of rhGH therapy, the level of FT4 was $1.19 \pm 0.18 \mathrm{ng} / \mathrm{dL}$, which was lower than the baseline value $(1.21 \pm 0.14 \mathrm{ng} / \mathrm{dL})$, but the difference was not statistically significant $(\mathrm{P}>0.05)$. At the $12^{\text {th }}, 18^{\text {th }}$ and $24^{\text {th }}$ month, the level of FT4 continued to fall $(1.18 \pm 0.18$, $1.16 \pm 0.15$ and $1.12 \pm 0.17 \mathrm{ng} / \mathrm{dL}$, respectively), and the difference was statistically significant $(\mathrm{P}=0.041, \mathrm{P}=0.044$ and $\mathrm{P}=0.013$, respectively).

\section{A comparison of the thyroid function of the puberty group and pre-puberty group of pediatric patients with GHD before and during the course of rbGH therapy}

The 55 patients were divided into two groups according to the development of their secondary sexual characteristics at baseline: a pre-puberty group and a puberty group. Forty-four pediatric patients were assigned to the prepuberty group, and these patients had an average age of $8.36 \pm 2.21$ years old. Eleven pediatric patients were assigned to the puberty group, and their average age was $11.50 \pm 1.53$ years old. As presented in Table 3, the thyroid functions of patients in these two groups were all within the normal range at baseline, while the TSH level of patients in the pre-puberty group was $1.91 \pm 0.62 \mathrm{mIU} / \mathrm{L}$, which was higher than that of patients in the puberty group $(1.63 \pm 0.54 \mathrm{mIU} / \mathrm{L})$, but the difference was not statistically significant $(\mathrm{P}=0.178)$. The level of FT4 in the puberty 
Table 3 The changes in TSH and $\mathrm{FT}_{4}$ levels in pediatric patients with GHD at baseline, and during the course of rhGH replacement therapy, between the puberty group and pre-puberty group

\begin{tabular}{|c|c|c|}
\hline Items & Pre-puberty group & Puberty group \\
\hline \multicolumn{3}{|l|}{ Baseline } \\
\hline Cases & 44 & 11 \\
\hline TSH (mIU/L) & $1.91 \pm 0.62$ & $1.63 \pm 0.54$ \\
\hline $\mathrm{FT}_{4}(\mathrm{ng} / \mathrm{dL})$ & $1.21 \pm 0.14$ & $1.24 \pm 0.14$ \\
\hline \multicolumn{3}{|c|}{ After 6-month treatment } \\
\hline Cases & 44 & 11 \\
\hline TSH (mIU/L) & $2.47 \pm 1.00^{\star \star}$ & $1.96 \pm 0.78$ \\
\hline $\mathrm{FT}_{4}(\mathrm{ng} / \mathrm{dL})$ & $1.19 \pm 0.19$ & $1.23 \pm 0.12$ \\
\hline \multicolumn{3}{|c|}{ After 12-month treatment } \\
\hline Cases & 35 & 9 \\
\hline TSH (mlU/L) & $2.15 \pm 0.76^{\star}$ & $1.79 \pm 0.79$ \\
\hline $\mathrm{FT}_{4}(\mathrm{ng} / \mathrm{dL})$ & $1.18 \pm 0.19$ & $1.17 \pm 0.16^{\star}$ \\
\hline \multicolumn{3}{|c|}{ After 18-month treatment } \\
\hline Cases & 28 & 4 \\
\hline TSH (mIU/L) & $2.42 \pm 0.72^{\star \star}$ & $1.36 \pm 0.24$ \\
\hline $\mathrm{FT}_{4}(\mathrm{ng} / \mathrm{dL})$ & $1.15 \pm 0.15$ & $1.25 \pm 0.09$ \\
\hline \multicolumn{3}{|c|}{ After 24-month treatment } \\
\hline Cases & 13 & 3 \\
\hline TSH (mIU/L) & $2.36 \pm 0.99^{*}$ & $1.99 \pm 0.45$ \\
\hline $\mathrm{FT}_{4}(\mathrm{ng} / \mathrm{dL})$ & $1.13 \pm 0.17^{\star}$ & $1.11 \pm 0.16$ \\
\hline
\end{tabular}

*compared after treatment with the baseline, there was statistical significance $(\mathrm{P}<0.05)$; ${ }^{*}$ compared after treatment with the baseline, there was significant difference $(P<0.01)$.

group was $1.24 \pm 0.14 \mathrm{ng} / \mathrm{dL}$, which was higher than that in the pre-puberty group $(1.21 \pm 0.14 \mathrm{ng} / \mathrm{dL})$, but the difference was also not statistically significant $(\mathrm{P}=0.465)$.

As illustrated in Table 3, pediatric patients in the prepuberty group exhibited a significant increase in TSH after six months of rhGH therapy, and it remained higher than that at the baseline level at the $12^{\text {th }}, 18^{\text {th }}$ and $24^{\text {th }}$ month of rhGH therapy. However, the levels of FT4 continued to fall during the course of therapy when compared to the baseline level and had significantly dropped after 24 months of therapy. In the puberty group, the levels of FT4 had only fallen at the $12^{\text {th }}$ month of therapy, but there were no significant changes in the levels of FT4 and TSH at the remaining observation time points.

\section{Discussion}

It was found that during the course of the rhGH therapy, none of the enrolled pediatric patients had FT4 levels lower than the lower limit of a normal FT4. Three pediatric patients in the pre-puberty group had a transient TSH value higher than the upper normal limit at the $6^{\text {th }}, 12^{\text {th }}$ and $18^{\text {th }}$ month, but this returned to normal. Compared with the levels at baseline, the TSH levels of the patients were significantly higher at the $6^{\text {th }}$ month of rhGH therapy and remained significantly higher at the $12^{\text {th }}, 18^{\text {th }}$ and $24^{\text {th }}$ month of therapy. Although the TSH and FT4 levels of pediatric patients in the present study increased during the treatment period, the time points were different from those reported in the relevant literature, which may be related to the dose of the GH therapy, the number of pediatric patients, and the individual differences of the patients. The investigators intend to expand the sample size, include the detection and analysis of FT3 levels, and explore the changes in thyroid hormone in pediatric patients according to their puberty status, in the future, in order to better understand the changes in thyroid function in pediatric patients with GHD during rhGH therapy.

In 2003, Giavoli et al. (8) conducted a 12-month follow-up study of rhGH therapy in 20 pediatric patients with idiopathic GHD (IGHD), but with normal thyroid function, and six pediatric patients with multiple pituitary hormone deficiencies (MPHD); four patients had central hypothyroidism and received levothyroxine (L-T4) replacement therapy. The results revealed that during the therapy, the levels of FT4 in both groups fell significantly $(\mathrm{P}<0.01)$, and the levels of FT4 in four pediatric patients with MPHD dropped to the level of hypothyroidism. However, there was no statistical significance in the changes in TSH level. Zheng (9) investigated the changes in thyroid function in 72 pediatric patients with GHD, who were treated with rhGH for six months in 2016. The average age of the patients was $10.3 \pm 2.4$ years old. The results showed that the TSH and FT4 levels of the patients had fallen slightly after three months of treatment. After six months of therapy, the two levels showed no significant changes when compared with those at baseline, and these above changes were not statistically significant.

In the present study, pediatric patients with GHD were divided into two groups, according to their puberty development stage at baseline: a pre-puberty group and a puberty group. The thyroid functions of the patients in these two groups were within the normal range at baseline. 
The TSH level of patients in the pre-puberty group was slightly higher than that of patients in the puberty group $(1.91 \pm 0.62 v s .1 .63 \pm 0.54 \mathrm{mIU} / \mathrm{L})$, and the FT4 level of patients in the puberty group was slightly higher than that of patients in the pre-puberty group $(1.24 \pm 0.14 \mathrm{vs}$. $1.21 \pm 0.14 \mathrm{ng} / \mathrm{dL})$. However, these differences were not statistically significant $(\mathrm{P}=0.178$ and $\mathrm{P}=0.465$, respectively).

The above results were not consistent with those reported in the relevant literature. This was considered to be related to the differences in study subjects, the number of patients, and the individual differences of the patients. $\mathrm{Wu}$ et al. (10) divided pediatric patients with short stature into two groups, according to their puberty status: a developed group and an undeveloped group. There were 104 pediatric patients in each group, 61 males and 43 females. Their results showed the level of FT3 in the developed group was significantly higher than in the undeveloped group, and the difference was statistically significant. The level of FT4 of the developed group was slightly lower than that of the undeveloped group, and the level of TSH of the male patients in the developed group was slightly lower than that of male patients in the undeveloped group, but there was no significant difference in the level of FT4 and TSH in female patients. In a study conducted by Marwaha et al. (11), the thyroid hormone levels of 1,021 boys (5-18 years old) and 1,113 girls (5-19 years old) in India were compared, according to gender and the stage of development. The results revealed that the level of FT3 rose with puberty, and this remained unchanged or slightly decreased after Tanner stage III, while the level of FT4 fell with puberty, and remained relatively stable from Tanner stage III onwards. The TSH levels in boys dropped at puberty, but remained basically unchanged in girls.

Changes in thyroid hormone levels during the treatment of IGHD were also observed in the puberty group and prepuberty group in the present study. Our results indicated that the level of TSH in these two groups had significantly increased $(\mathrm{P}=0.000)$ at six months after the beginning of rhGH therapy, and remained higher at 12, 18 and 24 months $(\mathrm{P}=0.047, \mathrm{P}=0.002$ and $\mathrm{P}=0.036$, respectively) of therapy, when compared to the level at baseline. The level of FT4 continuously fell during the course of therapy, when compared to the level at baseline, and this was statistically significant after 24 months of treatment $(\mathrm{P}=0.046)$. The level of FT4 in the puberty group was significantly lower than that at baseline only at the $12^{\text {th }}$ month of therapy $(\mathrm{P}=0.044)$, but there was no significant change in the levels of FT4 and TSH at the remaining observation time points of the therapy.

In 2005, Seminara et al. (12) conducted a 24-month follow-up study of rhGH therapy in 19 pre-puberty pediatric patients with IGHD. Their results showed that after 12 months of rhGH treatment, the level of FT4 had slightly decreased, but it had significantly increased after 24 months of therapy. However, the level of TSH did not significantly change during the treatment. In 2018, Witkowska-Sędek E et al. (13) investigated the changes in thyroid function in 117 pediatric patients with IGHD during 1-4 years of rhGH therapy. The average age of the patients was $9.8 \pm 3.5$ years old. They were grouped according to their pre-treatment puberty status. The results showed significant changes in TSH and FT4 levels in pre-puberty pediatric patients after rhGH therapy. When compared to pre-treatment, the TSH level had slightly increased after six months of rhGH therapy, but the difference was not statistically significant. The TSH level had significantly increased $(\mathrm{P}<0.01)$ after one year of treatment, but the TSH level had no significant change after another year of treatment. The level of FT4 had significantly decreased at six months of rhGH therapy $(\mathrm{P}<0.01)$, and remained lower than that before the treatment at one year $(\mathrm{P}<0.01)$ and two years of therapy $(\mathrm{P}<0.01)$. However, there was no significant change in the TSH and FT4 levels of the puberty pediatric patients when compared to the levels before treatment.

At present, there are differences in experimental and clinical research results regarding the effects of rhGH therapy on thyroid function in pediatric patients with GHD. This is likely to be due to the differences in the diagnostic criteria of GHD, the inclusion and exclusion criteria, the duration of GH therapy, the dose of the GH therapy, and the hormone measurement methods and research designs of different studies. Some studies have reported that rhGH therapy can significantly reduce the concentration of TT4 and FT4 (14-17) and significantly increase the level of triiodothyronine (T3) (14-16). Furthermore, some studies have indicated no significant changes in thyroid hormone concentration during the rhGH therapy (18). In addition, the concentration of TSH during the course of therapy might not change $(7,8,12,15)$, or it may decrease $(19)$. According to the related literature, the possible causes of the changes in thyroid function in pediatric patients with GHD during rhGH therapy might be the following: (I) at the peripheral level, GH mediates the increased extrathyroid transformation of $\mathrm{T} 4$ to $\mathrm{T} 3$. This is supported by research 
results that have revealed that during rhGH therapy, the level of anti-T3 decreases and/or the T3/T4 ratio increases (19-24). The monoiodization (activation pathway) of T4 to T3 is co-catalyzed by deiodinase type 1 (D1) and deiodinase type 2 (D2), while the inactivation pathway of T4 to RT3 is mediated by deiodinase type 3 (D3). The human GH receptor is expressed in many organs and tissues, and one or more deiodinases are mainly expressed in the following organs or tissues: D1 in the liver and kidneys; D2 in the bone and muscle; and, D1, D2 and D3 in the brain. For untreated pediatric patients with GHD, the transformation of T4 to T3 decreases, while RT3 increases (7). This suggests that endogenous GH plays a physiological role in the peripheral metabolism of thyroid hormones. However, the activation pathway controlled by GH in the human body remains to be confirmed; (II) at the central level, due to the increase in peripheral deiodination from T4 to T3 (20-22,24-26), the secretion of somatostatin increases, or the release of TSH is inhibited through the negative feedback mechanism of T3 in the thyroid gland $(3,27)$; (III) in addition to the deficiency in $\mathrm{GH}$, central hypothyroidism also occurs in pediatric patients with MPHD; and, (IV) there are signs of latent hypothyroidism of the hypothalamus in pediatric patients before treatment (28).

\section{Conclusions}

GH replacement therapy may affect the metabolism of thyroid hormones in pediatric patients with GHD. Meanwhile, compared with puberty IGHD pediatric patients, pre-puberty pediatric patients may be more likely to exhibit changes in thyroid hormone levels during rhGH therapy. Furthermore, it is necessary to regularly monitor the changes in thyroid function during the course of rhGH therapy, in order to identify and correct any abnormal thyroid function in the early stages and maximize the effect of the clinical treatment.

\section{Acknowledgments}

Funding: This study was supported by Research on optimal monitoring and treatment of type 1 diabetes and the promotion of key new technologies (No. 2017YFC1309601).

\section{Footnote}

Reporting Checklist: The authors have completed the MDAR checklist. Available at http://dx.doi.org/10.21037/tp-20-401

Data Sharing Statement: Available at http://dx.doi. org/10.21037/tp-20-401

Conflicts of Interest: All authors have completed the ICMJE uniform disclosure form (available at http://dx.doi. org/10.21037/tp-20-401). The authors have no conflicts of interest to declare.

Ethical Statement: The authors are accountable for all aspects of the work in ensuring that questions related to the accuracy or integrity of any part of the work are appropriately investigated and resolved. The study was conducted in accordance with the Declaration of Helsinki (as revised in 2013). The study was approved by the Ethics Committee of Tongji Hospital (NO.: 2014-120) and informed consent was taken from all the patients.

Open Access Statement: This is an Open Access article distributed in accordance with the Creative Commons Attribution-NonCommercial-NoDerivs 4.0 International License (CC BY-NC-ND 4.0), which permits the noncommercial replication and distribution of the article with the strict proviso that no changes or edits are made and the original work is properly cited (including links to both the formal publication through the relevant DOI and the license). See: https://creativecommons.org/licenses/by-nc-nd/4.0/.

\section{References}

1. Bell J, Parker KL, Swinford RD, et al. Long-term safety of recombinant human growth hormone in children. J Clin Endocrinol Metab 2010;95:167-77.

2. Hou L, Luo XP, Du ML, et al. Efficacy and safety of recombinant human growth hormone solution in children with growth hormone deficiency in China: A multicenter trial. Zhonghua Er Ke Za Zhi 2009;47:48-52.

3. Behan LA, Monson JP, Agha A. The interaction between growth hormone and the thyroid axis in hypopituitary patients. Clin Endocrinol (Oxf) 2011;74:281-8.

4. Akin F, Yaylali GF, Turgut S, et al. Growth hormone/ insulin like growth factor axis in patients with subclinical thyroid dysfunction. Growth Horm IGF Res 2009;19:252-5.

5. Laron Z. Interactions between the thyroid hormones and the hormones of the growth hormone axis. Pediatr Endocrinol Rev 2003;1 Suppl 2:244-9-discussion 250. 
6. Portes ES, Oliveira JHA, MacCagnan P, et al. Changes in serum thyroid hormones levels and their mechanisms during long-term growth hormone $(\mathrm{GH})$ replacement therapy in $\mathrm{GH}$ deficient children. Clin Endocrinol (Oxf) 2000;53:183-9.

7. Akin F, Yaylali GF, Turgut S, et al. Growth hormone/ insulin like growth factor axis in patients with subclinical thyroid dysfunction. Growth Horm IGF Res 2009; 19:252-5.

8. Giavoli C, Porretti S, Ferrante E, et al. Recombinant hGH replacement therapy and the hypothalamus-pituitarythyroid axis in children with GH deficiency: when should we be concerned about the occurrence of central hypothyroidism? Clin Endocrinol (Oxf) 2003;59:806-10.

9. Zheng CR, Ban B, Zhang M, et al. Effect of rhGH on thyroid function and its clinical significance in children with growth hormone deficiency. Chin J Child Health Care 2016;24:616-8.

10. Wu S, Liu QQ, Gu W, et al. The Correlation between Short Stature in Children and Adolescent Hormone Levels and BMI in Puberty. Genom Appl Biol 2017;36:3322-7.

11. Marwaha RK, Tandon N, Desai AK, et al. The evolution of thyroid function with puberty. Clin Endocrinol (Oxf) 2012;76:899-904.

12. Seminara S, Stagi S, Candura L, et al. Changes of thyroid function during long term hGH therapy in GHD children. A possible relationship with catch up growth? Horm Metab Res 2005;37:751-6.

13. Witkowska-Sędek E, Borowiec A, Majcher A, et al. Thyroid function in children with growth hormone deficiency during long-term growth hormone replacement therapy. Cent Eur J Immunol 2018;43:255-61.

14. Glynn N, Kenny H, Salim T, et al. Alterations in thyroid hormone levels following growth hormone replacement exert complex biological effects. Endocr Pract 2018;24:342-50.

15. Jørgensen JO, Pedersen SB, Børglum J, et al. Fuel metabolism, energy expenditure, and thyroid function in growth hormone-treated obese women: a double-blind placebo-controlled study. Metabolism 1994;43:872-7.

16. Glynn N, Kenny H, Quisenberry L, et al. The effect of growth hormone replacement on the thyroid axis in patients with hypopituitarism: in vivo and ex vivo studies. Clin Endocrinol (Oxf) 2017;86:747-54.

17. Keskin M, Bayramoglu E, Aycan Z. Effects of 1-year growth hormone replacement therapy on thyroid volume and function of the children and adolescents with idiopathic growth hormone deficiency. J Pediatr Endocrinol Metab 2017;30:1187-90.

18. Rose SR, Leong GM, Yanovski JA, et al. Thyroid function in non-growth hormone deficient short children during a placebo-controlled double blind trial of recombinant growth hormone therapy. J Clin Endocrinol Metab 1995;80:320-4.

19. Grunfeld C, Sherman BM, Cavalieri RR. The acute effects of human growth hormone administration on thyroid function in normal men. J Clin Endocrinol Metab 1988;67:1111-4.

20. Porter BA, Refetoff S, Rosenfeld RL, et al. Abnormal thyroxine metabolism in hyposomatotrophic dwarfism and inhibition of responsiveness to TRH during GH therapy. Pediatrics 1973;51:668-74.

21. Sato T, Suzukui Y, Taketani T, et al. Enhanced peripheral conversion of thyroxine to triiodothyronine during hGH therapy in GH deficient children. J Clin Endocrinol Metab 1977;45:324-9.

22. Rezvani I, DiGeorge AM, Dowshen SA, et al. Action of human growth hormone (hGH) on extrathyroidal conversion of thyroxine (T4) to triidothironine (T3) in children with hypothyroidism. Pediatr Res 1981;15:6-9.

23. Wyatt DT, Gesundheit N, Sherman B. Changes in thyroid hormones levels during growth hormone therapy in initially euthyroid patients: lack of need for thyroxine supplementation. J Clin Endocrinol Metab 1998;83:3493-7.

24. Jørgensen JO, Pedersen SA, Laurberg P, et al. Effects of Growth Hormone Therapy on Thyroid Function of Growth Hormone-Deficient Adults with and without Concomitant Thyroxine-Substituted Central Hypothyroidism. J Clin Endocrinol Metab 1989;69:1127-32.

25. Oliner L, Ballantine JJ. Effect of human growth hormone on thyroidal secretion, radiothyroxine turnover and transport in man. J Clin Endocrinol Metab 1968;28:603-7.

26. Cobb WE, Reichlin S, Jackson IM. Growth hormone secretory status is a determinant of the thyrotropin response to thyrotropin-releasing hormone in euthyroid patients with hypothalamic-pituitary disease. J Clin Endocrinol Metab 1981;52:324-9.

27. Jørgensen JO, Møller J, Laursen T, et al. Growth hormone administration stimulates energy expenditure and extrathyroidal conversion of thyroxine to triiodothyronine 
in a dose-dependent manner and suppresses circadian thyrotrophin levels: studies in GH-deficient adults. Clin Endocrinol (Oxf) 1994;41:609-14.

28. Hara T, Tamai H, Mukuta T, et al. Triphasic changes in

Cite this article as: Yao Q, Zheng D, Liang Y, Hou L, Ying YQ, Luo XP, Wu W. The effects of recombinant human growth hormone therapy on thyroid function in pediatric patients with growth hormone deficiency. Transl Pediatr 2021;10(4):851-859. doi: $10.21037 /$ tp-20-401 thyroid function in a patient with primary hypothyroidism in the course of pregnancy and the early post-partum period. Endocr J 1997;44:581-7. 